\title{
DENSITY THEOREMS FOR SAMPLING AND INTERPOLATION IN THE BARGMANN-FOCK SPACE
}

\author{
KRISTIAN SEIP
}

\begin{abstract}
We give a complete description of sampling and interpolation in the Bargmann-Fock space, based on a density concept of Beurling. Roughly speaking, a discrete set is a set of sampling if and only if its density in every part of the plane is strictly larger than that of the von Neumann lattice, and similarly, a discrete set is a set of interpolation if and only if its density in every part of the plane is strictly smaller than that of the von Neumann lattice.
\end{abstract}

\section{INTRODUCTION AND RESULTS}

The work presented in this announcement is based on Beurling's lectures on balayage of Fourier-Stieltjes transforms and interpolation for an interval on $\mathbf{R}^{1}$ [3]. We observe that Beurling's problems concerning functions of exponential type have natural counterparts for functions of order two, finite type and find that, indeed, so have his main results. The most interesting part, however, is that Beurling's ideas are applicable also in the Hilbert space setting, yielding a complete description of sampling and interpolation in the Bargmann-Fock space. The simplicity of these results is quite remarkable when compared to the situation in the Paley-Wiener space (the corresponding Hilbert space of functions of exponential type) and to the extensive literature on nonharmonic Fourier series and, in particular, Riesz bases of complex exponentials [20].

This research is motivated by a recent development in signal analysis and applied mathematics, which was initiated by Daubechies, Grossmann, and Meyer $[5,4,6]$. Their work inspired us to search for a general characterization of the information needed to represent signals, as functions in the Bargmann-Fock space. Our results can be seen as sharp statements about the Nyquist density and its meaning in this context.

In order to describe more precisely the problems to be considered, a few definitions are needed. For $\alpha>0$, let $d \mu_{\alpha}(z)=(\alpha / \pi) e^{-\alpha|z|^{2}} d x d y, z=$ $x+i y$, and define the Bargmann-Fock space $F_{\alpha}^{2}$ to be the collection of entire functions $f(z)$ for which

$$
\|f\|_{2}=\|f\|_{\alpha, 2}=\int_{\mathbf{C}}|f(z)|^{2} d \mu_{\alpha}(z)<\infty .
$$

Received by the editors August 9, 1991 and, in revised form, November 19, 1991.

1991 Mathematics Subject Classification. Primary 30D10, 30E05, 46E20; Secondary 81D30. 
$F_{\alpha}^{2}$ is a Hilbert space with reproducing kernel $K(z, \zeta)=e^{\alpha \bar{z} \zeta}$; i.e., for every $f \in F_{\alpha}^{2}$ we have

$$
f(z)=\langle f, K(z, \cdot)\rangle=\int_{\mathbf{C}} f(\zeta) \overline{K(z, \zeta)} d \mu_{\alpha}(\zeta)
$$

The normalized reproducing kernels, $k_{\zeta}(z)=K(\zeta, \zeta)^{-1 / 2} K(\zeta, z)$, can be viewed as the natural (well-localized) building blocks of $F_{\alpha}^{2}$. They correspond, via the Bargmann transform, to the canonical coherent states of quantum mechanics and to Gabor wavelets in signal analysis. This relation is the reason for the importance of the Bargmann-Fock space; see [8] for general information and [5] for more background on the problems treated here.

We say that a discrete set $\Gamma$ of complex numbers is a set of sampling for $F_{\alpha}^{2}$ if there exist positive numbers $A$ and $B$ such that

$$
A\|f\|_{2}^{2} \leq \sum_{z \in \Gamma} e^{-\alpha|z|^{2}}|f(z)|^{2} \leq B\|f\|_{2}^{2}
$$

for all $f \in F_{\alpha}^{2}$. If to every $l^{2}$-sequence $\left\{a_{j}\right\}$ of complex numbers there exists an $f \in F_{\alpha}^{2}$ such that $e^{-\alpha\left|z_{j}\right|^{2} / 2} f\left(z_{j}\right)=a_{j}$ for all $j$, then $\Gamma=\left\{z_{j}\right\}$ is said to be a set of interpolation for $F_{\alpha}^{2}$. A set of sampling corresponds, in the terminology of [7], to a frame of coherent states. A set of both sampling and interpolation (which does not exist) would correspond to a Riesz basis of coherent states.

With a view to applications in physics and signal analysis, Daubechies and Grossmann posed the problem of finding the lattices $z_{m n}=m a+i n b, m, n \in$ $\mathbf{Z}$, that are sets of sampling [5]. They proved that a lattice could be a set of sampling only if $a b<\pi / \alpha$ and conjectured this condition also to be sufficient. For $a b=\pi /(\alpha N), N$ an integer $\geq 2$, they found (1) to hold by providing explicit expressions for the optimal constants $A, B$. Daubechies was later able to show that a lattice is a set of sampling whenever $N^{-1}<0.996$ [4].

We prove that the density criterion of the Daubechies-Grossmann conjecture applies not only to lattices, but to arbitrary discrete sets. We should add here that the conjecture was proved independently by Lyubarskii [12] and by Wallstén and the author [19].

For the description to be given of sets of sampling and interpolation, we need Beurling's density concept as generalized by Landau [11]. We consider then uniformly discrete sets, i.e., discrete sets $\Gamma=\left\{z_{j}\right\}$ for which $q=\inf _{j \neq k}\left|z_{j}-z_{k}\right|$ $>0$. We fix a compact set $I$ of measure 1 in the complex plane, whose boundary has measure 0 . Let $n^{-}(r)$ and $n^{+}(r)$ denote, respectively, the smallest and largest number of points from $\Gamma$ to be found in a translate of $r I$. We define the lower and upper uniform densities of $\Gamma$ to be

$$
D^{-}(\Gamma)=\liminf _{r \rightarrow \infty} \frac{n^{-}(r)}{r^{2}} \quad \text { and } D^{+}(\Gamma)=\limsup _{r \rightarrow \infty} \frac{n^{+}(r)}{r^{2}}
$$

respectively. It was proved by Landau that these limits are independent of $I$. 
Our main theorems are the following (the sufficiency part of the theorems were obtained in collaboration with Wallstén [19].

Theorem 1.1. $A$ discrete set $\Gamma$ is a set of sampling for $F_{\alpha}^{2}$ if and only if it can be expressed as a finite union of uniformly discrete sets and contains a uniformly discrete subset $\Gamma^{\prime}$ for which $D^{-}\left(\Gamma^{\prime}\right)>\alpha / \pi$.

Theorem 1.2. A discrete set $\Gamma$ is a set of interpolation for $F_{\alpha}^{2}$ if and only if it is uniformly discrete and $D^{+}(\Gamma)<\alpha / \pi$.

Remark 1. Decomposition and interpolation theorems for general discrete sets were obtained in [10], however, without any indication of a critical density. The results in [10] appear as part of a certain trend in harmonic analysis, and the analogy to the theory of nonharmonic Fourier series does not seem to have been realized.

Remark 2. The lattice with $a=b=\sqrt{\pi / \alpha}$ is called the von Neumann lattice, since von Neumann claimed (without proof) that it is a set of uniqueness [13]; many proofs have later been given [2, 14, 1, 19]. See [9] for an attempted repair of the "defect" of the von Neumann lattice that it is neither a set of sampling nor one of interpolation.

We consider also the analogues in our setting of the problems treated in [3]. We introduce then the Banach space $F_{\alpha}^{\infty}$, consisting of those entire functions $f(z)$ for which

$$
\|f\|_{\infty}=\|f\|_{\alpha, \infty}=\sup _{z} e^{-\alpha|z|^{2} / 2}|f(z)|<\infty .
$$

$\Gamma$ is said to be a set of sampling for $F_{\alpha}^{\infty}$ if there exists a positive number $K$ such that

$$
\|f\|_{\infty} \leq K \sup _{z \in \Gamma} e^{-\alpha|z|^{2} / 2}|f(z)|
$$

for all $f \in F_{\alpha}^{\infty}$. If to every bounded sequence $\left\{a_{j}\right\}$ of complex numbers there exists an $f \in F_{\alpha}^{\infty}$ such that $e^{-\alpha\left|z_{j}\right|^{2} / 2} f\left(z_{j}\right)=a_{j}$ for all $j$, we say that $\Gamma=\left\{z_{j}\right\}$ is a set of interpolation for $F_{\alpha}^{\infty}$. We have then the following counterparts of Beurling's two density theorems in [3] (we are using the term sampling instead of balayage as in [3], which seems natural since we no longer have the relation to Fourier-Stieltjes transforms).

Theorem 1.3. $A$ discrete set $\Gamma$ is a set of sampling for $F_{\alpha}^{\infty}$ if and only if it contains a uniformly discrete subset $\Gamma^{\prime}$ for which $D^{-}\left(\Gamma^{\prime}\right)>\alpha / \pi$.

Theorem 1.4. A discrete set $\Gamma$ is a set of interpolation for $F_{\alpha}^{\infty}$ if and only if it is uniformly discrete and $D^{+}(\Gamma)<\alpha / \pi$.

Let us remark, as Beurling did, that the problems and some of the results extend to several variables. We would also like to mention the following interesting question: What are the corresponding density theorems for weighted Bergman spaces? See $[16,18]$ for a treatment of this problem. 


\section{THE NECESSITY PARTS OF THE THEOREMS-INDICATION OF PROOF}

In this section we make a few remarks to indicate how to prove the necessity parts of the theorems. Details are given in [17]. When unspecified, $p$ is taken to be either 2 or $\infty$.

We remark first that the translations

$$
\left(T_{a} f\right)(z)=e^{\alpha \bar{a} z-\alpha|a|^{2} / 2} f(z-a)
$$

act isometrically in $F_{\alpha}^{p}$. This translation invariance implies immediately that $\Gamma+z$ is a set of sampling (interpolation) if and only if $\Gamma$ is a set of sampling (interpolation) and it permits us to translate our analysis around an arbitrary point $z$ to 0 .

Another important feature of $F_{\alpha}^{p}$ is the following compactness property: If $\left\{f_{n}\right\}$ is a sequence in the ball

$$
\left\{f \in F_{\alpha}^{p}:\|f\|_{p} \leq R\right\}
$$

then there is a subsequence $\left\{f_{n_{k}}\right\}$ converging pointwise and uniformly on compact sets to some function in the ball. This is immediate from the definition of $F_{\alpha}^{p}$ and a normal family argument.

Following Beurling, for a closed set $\Gamma$, we let $W(\Gamma)$ denote the collection of weak limits of translates $\Gamma+z[3$, p. 344]. The compactness property and the translation invariance of $F_{\alpha}^{p}$ make $W(\Gamma)$ a crucial tool in our analysis. Indeed, it turns out that all of Beurling's arguments concerning $W(\Gamma)$ can be carried over to our situation.

Most of the work needed to prove the necessity parts of Theorems 1.3 and 1.4 consists in transferring Beurling's arguments. In addition to the ingredients mentioned above, a simple substitute for Bernstein's theorem is used. Moreover, adapting an idea of Landau [11], we make use, at a certain stage in the proof of Theorem 1.3, of the nice properties of the normalized monomials (normalized in $F_{\alpha}^{2}$ ), see $[8$, p. $39 ; 15]$.

For the $L^{2}$ problem, the basic auxiliary result is the following lemma.

Lemma 2.1. There is no discrete subset of $C$ that is both a set of sampling and a set of interpolation for $F_{\alpha}^{2}$.

This lemma has the following consequences.

Lemma 2.2. If $\Gamma$ is a set of sampling for $F_{\alpha}^{2}$, then so is $\Gamma \backslash\{\zeta\}$ for any $\zeta \in \Gamma$.

Lemma 2.3. If $\Gamma$ is a set of interpolation for $F_{\alpha}^{2}$, then so is $\Gamma \cup\{\zeta\}$ for any $\zeta \notin \Gamma$.

The main difficulty in proving the necessity part of Theorem 1.1 consists in showing that $D^{-}(\Gamma)>\alpha / \pi$ if $\Gamma$ is uniformly discrete and a set of sampling. This problem can now be dealt with in the following way. Consider such a $\Gamma$. It is easy to show that $W(\Gamma)$ consists only of sets of sampling. By Lemma 2.2 we have that every set of sampling for $F_{\alpha}^{2}$ is a set of uniqueness for $F_{\alpha}^{\infty}$. For suppose $\Gamma_{0}$ is a set of sampling for $F_{\alpha}^{2}$ and that $g \in F_{\alpha}^{\infty}$ vanishes on $\Gamma_{0}$. 
Then the function

$$
f(z)=g(z) /\left(z-z_{1}\right)\left(z-z_{2}\right),
$$

$z_{1}, z_{2} \in \Gamma_{0}$, belongs to $F_{\alpha}^{2}$ and vanishes on $\Gamma_{0} \backslash\left\{z_{1}, z_{2}\right\}$. This contradicts Lemma 2.2.

Thus every set in $W(\Gamma)$ is a set of uniqueness for $F_{\alpha}^{\infty}$. It can be proved that $\Gamma$ is a set of sampling for $F_{\alpha}^{\infty}$ if and only if every $\Gamma_{0} \in W(\Gamma)$ is a set of uniqueness for $F_{\alpha}^{\infty}$ (see Theorem 3 in [3, p. 345]). Hence by Theorem 1.3, $D^{-}(\Gamma)>\alpha / \pi$.

As to the necessity part of Theorem 1.2, we remark that Lemma 2.3 enables us to carry over Beurling's technique used for the corresponding $L^{\infty}$ problem; here a slight modification of the key notion ' $\rho(z ; \Gamma)$ ' is needed, see [3, p. 352].

\section{THE SUFFICIENCY PARTS OF THE THEOREMS-INDICATION OF PROOF}

Details can be found in [19].

Let $\Lambda=\left\{\lambda_{m n}\right\}$ denote a square lattice; that is, $\lambda_{m n}=\sqrt{\pi / \alpha}(m+i n)$ for all integers $m, n$ and some positive number $\alpha . \alpha / \pi$ will be referred to as the density of $\Lambda$. We observe that the Weierstrass $\sigma$-function, $\sigma(z)$, associated to $\Lambda$ plays a role in the Bargmann-Fock space analogous to that of the sine in the Paley-Wiener space. This permits us to use techniques similar to some of those employed for functions of exponential type.

We introduce the analogues of $\sigma(z)$ for uniformly discrete sets that are close to a square lattice in the following sense. $\Gamma=\left\{z_{m n}\right\}$ is uniformly close to $\Lambda$ if there exists a positive number $Q$ such that $\left|z_{m n}-\lambda_{m n}\right| \leq Q$ for all $m$ and $n$.

To $\Gamma$, uniformly close to a square lattice, we associate a function $g(z)$ defined by

$$
g(z)=\left(z-z_{00}\right) \prod_{m, n}^{\prime}\left(1-\frac{z}{z_{m n}}\right) \exp \left(\frac{z}{z_{m n}}+\frac{1}{2} \frac{z^{2}}{\lambda_{m n}^{2}}\right)
$$

where $z_{00}$ is the point of $\Gamma$ closest to 0 . Using the quasi-periodicity of the $\sigma$-function, we obtain the following estimates on the growth of $g$.

Lemma 3.1. Let $\Gamma$ be uniformly close to the square lattice $\Lambda$ of density $\alpha / \pi$. Then there exist constants $C_{1}, C_{2}$ and $c$, depending only on $Q$ and $q$, such that for every $z$ we have

$$
\begin{gathered}
\left|e^{-\alpha|z|^{2} / 2} g(z)\right| \geq C_{1} e^{-c|z| \log |z|} \operatorname{dist}(z, \Gamma), \\
\left|e^{-\alpha|z|^{2} / 2} g(z)\right| \leq C_{2} e^{c|z| \log |z|},
\end{gathered}
$$

and for every $z_{m n} \in \Gamma$ we have

$$
\left|e^{-\alpha\left|z_{m n}\right|^{2} / 2} g^{\prime}\left(z_{m n}\right)\right| \geq C_{1} e^{-c\left|z_{m n}\right| \log \left|z_{m n}\right|} .
$$

By this lemma and the calculus of residues, we obtain the following Lagrangetype interpolation formula.

Lemma 3.2. Let $\Gamma=\left\{z_{m n}\right\}$ be uniformly close to the square lattice of density $\beta / \pi$, and let $g$ be the function associated to $\Gamma$ by (2). If $\alpha<\beta$ we have for 
each $f \in F_{\alpha}^{\infty}$

$$
f(z)=\sum_{m, n} \frac{f\left(z_{m n}\right)}{g^{\prime}\left(z_{m n}\right)} \frac{g(z)}{z-z_{m n}}
$$

with uniform convergence on compact sets.

The difficulty in proving the sufficiency part of Theorem 1.1 consists in verifying the left inequality in (1). We assume then, without loss of generality, that $\Gamma$ is uniformly discrete and uniformly close to the square lattice of density $\beta / \pi$, where $D^{-}(\Gamma)=\beta / \pi$; see $[3$, p. 356] for an argument justifying this claim. We write

$$
\int_{C}|f(z)|^{2} d \mu_{\alpha}(z)=\sum_{k, l} \int_{R}\left|\left(T_{\lambda_{k l}} f\right)(z)\right|^{2} d \mu_{\alpha}(z)
$$

where $R=\left\{z=x+i y:|x|<\frac{1}{2} \sqrt{1 / \alpha},|y|<\frac{1}{2} \sqrt{1 / \alpha}\right\}$. In order to estimate the summands on the right, we use Lemma 3.2 to write

$$
\left(T_{\lambda_{k l}} f\right)(z)=\sum_{m, n} \frac{\left(T_{\lambda_{k l}} f\right)\left(z_{m n}+\lambda_{k l}\right)}{g_{\lambda_{k l}}^{\prime}\left(z_{m n}+\lambda_{k l}\right)} \frac{g_{\lambda_{k l}}(z)}{z-z_{m n}-\lambda_{k l}},
$$

where $g_{\lambda_{k l}}$ is the function associated to $\Gamma+\lambda_{k l}$ by (2). Lemma 3.1 is used to estimate this expression, and after some computation we obtain the desired estimate.

The sufficiency part of Theorem 1.3 can be proved in the same way, or more easily, by Beurling's method [3, p. 346].

In order to prove the sufficiency parts of Theorems 1.2 and 1.4 , we note first that we may assume that $\Gamma$ is uniformly close to a square lattice of density $\beta / \pi$, where $D^{+}(\Gamma)=\beta / \pi[3$, p. 356]. The interpolation problem is then solved explicitly by the following formula,

$$
f(z)=\sum_{m, n} a_{m n} e^{\alpha \bar{z}_{m n} z-\alpha\left|z_{m n}\right|^{2}} \frac{g_{-z_{m n}}\left(z-z_{m n}\right)}{\left(z-z_{m n}\right)} .
$$

Lemma 3.1 is used to verify this assertion.

\section{REFERENCES}

1. H. Bacry, A. Grossmann, and J. Zak, Proof of the completeness of lattice states in the $k q$ representation, Phys. Rev. B 12 (1975), 1118-1120.

2. V. Bargmann, P. Butero, L. Girardello, and J. R. Klauder, On the completeness of coherent states, Rep. Mod. Phys. 2 (1971), 221-228.

3. A. Beurling, The collected works of Arne Beurling, vol. 2 Harmonic analysis (L. Carleson, P. Malliavin, J. Neuberger, and J. Wermer, eds.), Birkhäuser, Boston, MA, 1989, pp. 341365.

4. I. Daubechies, The wavelet transform, time-frequency localization and signal analysis, IEEE Trans. Inform. Theory 36 (1990), 961-1005.

5. I. Daubechies and A. Grossmann, Frames in the Bargmann space of entire functions, Comm. Pure Appl. Math., 41 (1988), 151-164.

6. I. Daubechies, A. Grossmann, and Y. Meyer, Painless nonorthogonal expansions, J. Math. Phys. 27 (1986), 1271-1283. 
7. R. J. Duffin and A. C. Schaeffer, A class of nonharmonic Fourier series, Trans. Amer. Math. Soc. 72 (1952), 341-366.

8. G. B. Folland, Harmonic analysis in phase space, Princeton Univ. Press, Princeton, NJ, 1989.

9. K. Gröchenig and D. Walnut, A Riesz basis for the Bargmann-Fock space related to sampling and interpolation, Ark. Mat. (to appear).

10. S. Janson, J. Peetre, and R. Rochberg, Hankel forms and the Fock space, Revista Mat. Iberoamer. 3 (1987), 61-138.

11. H. J. Landau, Necessary density conditions for sampling and interpolation of certain entire functions, Acta Math. 117 (1967), 37-52.

12. Y. Lyubarskii, Frames in the Bargmann space of entire functions, manuscript, 1990.

13. J. von Neumann, Foundations of quantum mechanics, Princeton Univ. Press, Princeton, NJ, 1955.

14. A. M. Perelomov, On the completeness of a system of coherent states, Theor. Math. Phys. 6 (1971), 156-164.

15. K. Seip, Reproducing formulas and double orthogonality in Bargmann and Bergman spaces, SIAM J. Math. Anal. 22 (1991), 856-876.

16. Regular sets of sampling and interpolation for weighted Bergman spaces, Proc. Amer. Math. Soc. (to appear).

17. Density theorems for sampling and interpolation in the Bargmann-Fock space I, J. Reine Angew. Math. (to appear).

18. K. Seip, Beurling type density theorems in the unit disk, manuscript, 1992.

19. K. Seip and R. Wallstén, Density theorems for sampling and interpolation in the BargmannFock space II, J. Reine Angew. Math. (to appear).

20. R. M. Young, An introduction to nonharmonic Fourier series, Academic Press, New York, 1980.

Division of Mathematical Sciences, Norwegian Institute of Technology, N-7034 TRONDHEIM-NTH, NORWAY

E-mail address: seip@imf.unit.no 